\author{
Abstracta Iranica \\ Abstracta Iranica Revue bibliographique pour le domaine irano-aryen \\ Volume 37-38-39 | 2018 \\ Comptes rendus des publications de 2014-2016
}

\title{
Nobuaki Kondo (ed.). Mapping Safavid Iran
}

\section{Maria Szuppe}

\section{OpenEdition}

\section{Journals}

Édition électronique

URL : http://journals.openedition.org/abstractairanica/46098

DOI : 10.4000/abstractairanica.46098

ISBN : 1961-960X

ISSN : 1961-960X

Éditeur :

CNRS (UMR 7528 Mondes iraniens et indiens), Éditions de l'IFRI

Référence électronique

Maria Szuppe, "Nobuaki Kondo (ed.). Mapping Safavid Iran », Abstracta Iranica [En ligne], Volume 37-38-39 | 2018, document 1, mis en ligne le 30 décembre 2018, consulté le 26 septembre 2020. URL : http://journals.openedition.org/abstractairanica/46098; DOI : https://doi.org/10.4000/ abstractairanica.46098

Ce document a été généré automatiquement le 26 septembre 2020.

Tous droits réservés 


\title{
Nobuaki Kondo (ed.). Mapping Safavid Iran
}

\author{
Maria Szuppe
}

\section{RÉFÉRENCE}

Nobuaki Kondo (ed.). Mapping Safavid Iran. Fuchu - Tokyo: Research Institute for Languages and Cultures of Asia and Africa (ILCAA), 2015, 246p., 6 illustrations n\&b, 10 illustrations couleurs, 1 carte, 5 tableaux, index général. (Studia Culturae Islamicae, 105 ; MEIS Series, 22), ISBN 978-4-86337-211-5

1 Ce volume collectif rassemble neuf contributions par d'éminents spécialistes, issues des travaux d'un colloque international tenu à Tokyo en 2013. Dans sa Préface (p. 1-5), Nobuaki Kondo positionne cette publication comme le premier ouvrage collectif «safavide» produit au Japon, qui, en cette qualité, reflète incontestablement l'importance prise dans ce pays par les recherches safavides durant le dernier quart du $\mathrm{XX}^{\mathrm{e}}$ siècle et le début du $\mathrm{XXI}{ }^{\mathrm{e}}$ siècle. En effet, des spécialistes japonais internationalement reconnus du domaine, eux-mêmes partiellement formés en France auprès de Jean Aubin et de Jean Calmard en particulier, ont été maîtres d'une nouvelle génération de chercheurs qui a déjà produit des contributions marquantes. De cette façon, N. Kondo replace également le présent volume dans la tradition des études safavides mondiales récentes, notamment celles des années 2000, qui s'intéressent particulièrement à l'Etat safavide dans le contexte large de l'histoire macro-régionale et, même, mondiale. Il conviendrait certainement d'ajouter ici que l'élan original ayant amené ce renouveau des études safavides remonte bien à l'organisation, sur l'initiative de J. Calmard, d'un premier "colloque safavide» international en 1989 à Paris (cf. J. Calmard, éd., Études safavides, Paris-Téhéran: IFRI, 1993 [Bibliothèque iranienne $\mathrm{n}^{\circ}$ 39]), colloque suivi d'une deuxième rencontre «safavide » à Cambridge en 1993 et une nouvelle publication collective (cf. C. Melville, Safavid Persia: History and Politics of an Islamic Society, London : I. B. Tauris, 1996 [Pembroke Papers, n 4]). 
2 Les neuf articles qui constituent le présent volume font émerger, grâce à leurs approches diversifiées et à une variété de sources mises à contribution, l'image d'un État multiforme par excellence - y compris dans sa composition ethnique et sociale - et essentiellement dynamique. Ainsi, on voit l'État safavide rester en interaction constante avec le monde environnant par ses activités diplomatiques et commerciales longue distance (jusqu'au Japon), par exemple, mais aussi par la faculté que manifeste sa société pour l'intégration, la transformation et, enfin, le développement spécifique des éléments de traditions politiques, religieuses et culturelles, que l'on reconnaît ensuite comme « éléments-clés » de la constitution de l'Iran moderne et contemporain.

3 Liste des articles : -Rula Jurdi Abisaab, «From Textual Evidence to Ijtihād: The Twelver Shi'a Juristic Tradition, 10th-16th Century » (P. 7-40); -Nobuaki Kondo, «The Shah 'Abd al-'Azim Shrine and its Vaqf under the Safavids» (P.41-65); -Rudi Matthee, "The Safavid King Who Was Crowned Twice: The Enthronement of Safi Mirza as Shah Safi II in 1077/1666, and as Shah Sulayman in 1078/1668" (P. 67-98); -Yukako Goto, «Development of Transport and Growth of Cultural Homogenization in the later Safavid Period » (P. 99-126) ; -Akihiko Yamaguchi, «The Safavid Legacy as Viewed from the Periphery: The Formation of Iran and Political Integration of a Kurdish Emirate " (P. 127-154) ; -Giorgio Rota, « Aq Qoyunlu and Safavid European Diplomacy: Strategy, Millenarism, Wishful Thinking» (P. 155-170); -Sholeh Quinn, «A Historian on the Move: An Early Modern Persian Chronicler under the Safavids and the Mughals » (P.171-188); -Sebouh David Aslanian, «Julfan Merchants and European East India Companies: Overland Trade, Protection Costs, and the Limits of Collective Selfrepresentation in Early Modern Safavid Iran » (P. 189-222); -Katsuhiko Abe, «Silk for the Court: Safavid Silk Textiles in Japanese Collections" (cf. cr dans ce volume) (p. 223-241).

\section{AUTEURS}

\section{MARIA SZUPPE}

CNRS, Mondes iranien et indien, Paris 